\title{
Impact de l'agriculture climato-intelligente sur les stocks de carbone organique du sol à Madagascar
}

\author{
Tantely Maminiaina Razafimbelo ${ }^{1, *}$, Andry Andriamananjara ${ }^{1}$, Tovonarivo Rafolisy ${ }^{1}$, Herintsitohaina \\ Razakamanarivo $^{1}$, Dominique Masse ${ }^{2}$, Eric Blanchart ${ }^{2}$, Marie-Virginie Falinirina ${ }^{1}$, Laetitia Bernard ${ }^{2}$, \\ Nasandratra Ravonjiarison ${ }^{1}$ et Alain Albrecht ${ }^{2}$ \\ ${ }^{1}$ Laboratoire des radio-isotopes, université d'Antananarivo, Antananarivo, Madagascar \\ ${ }^{2}$ UMR Eco\&Sols, Institut de recherche pour le développement (IRD), Univ Montpellier, Montpellier, France
}

\begin{abstract}
Résumé - L'agriculture climato-intelligente (Climate Smart Agriculture) vise à lutter contre le changement climatique et à s'y adapter tout en combattant l'insécurité alimentaire. À Madagascar, différentes pratiques agricoles ont été testées depuis une vingtaine d'années par des organisations non gouvernementales et des institutions de recherche afin d'accroître les rendements, maintenir la fertilité des sols et augmenter les revenus des ménages. Ces pratiques sont l'agriculture de conservation, l'agroforesterie et l'utilisation d'intrants ou d'amendements organiques comme le compost ou le fumier. Cette étude a pour objet de documenter les impacts de quelques pratiques d'agriculture climato-intelligente sur le stockage du carbone dans le sol. Les résultats montrent que le stock de carbone des sols varie fortement d'une pratique à l'autre. Pour l'agriculture de conservation, la différence de stock varie de 0 à $1,82 \mathrm{Mg} \mathrm{Cha}^{-1} \mathrm{an}^{-1}$ par rapport à la pratique traditionnelle (labour et exportation des résidus). Pour l'agroforesterie, la différence de stock entre des systèmes rizicoles sous girofliers et des pratiques de riziculture sur brûlis est de $0,68 \mathrm{Mg}$ $\mathrm{C} \mathrm{ha}^{-1} \mathrm{an}^{-1}$. L'utilisation d'apports organiques comme le fumier, le compost ou les déchets urbains a induit des augmentations de carbone du sol de $0,16,0,81$ et $0,42 \mathrm{Mg} \mathrm{Cha}^{-1} \mathrm{an}^{-1}$, mais les effets de ces apports organiques ne sont pas significatifs du fait de la très grande variabilité inter-parcellaire des stocks mesurés. Les pratiques d'agriculture climato-intelligente permettent ainsi d'augmenter la teneur en carbone du sol et ont donc des potentiels d'atténuation du changement climatique, mais ce potentiel est très variable selon la pratique considérée. Un éventuel impact à l'échelle du pays dépendra de l'étendue de l'adoption de ces différentes pratiques.
\end{abstract}

Mots-clés : adaptation / atténuation / changement climatique / matière organique / séquestration du carbone / Madagascar

\begin{abstract}
Climate smart agriculture impact on soil organic carbon stocks in Madagascar. Climate smart agriculture is presented as a solution to alleviate food insecurity, mitigate climate change and contribute to climate change adaptation. In Madagascar, different sustainable agricultural practices were implemented in the last 20 years by many non-governmental organizations in order to increase crop yields, maintain soil fertility and increase household income. These practices are conservation agriculture, agroforestry systems and the use of organic fertilizers as compost and manure. This study aimed to compare the soil organic carbon storage of some climate smart practices in the Malagasy context. The soil organic carbon stock of each practice, distributed in several sites among the island, was measured and compared with the traditional practices of each study site. For conservation agriculture, the differences in the soil

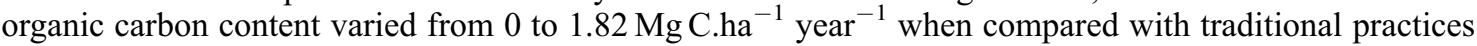
(tillage and crop residues exported). For agroforestry, a difference in the soil organic carbon content of $0.68 \mathrm{MgC} \mathrm{ha}^{-1}$ year $^{-1}$ was found when comparing with the slash and burn practices in the East Coast of Madagascar but no significant soil organic carbon content was found in the Malagasy Highlands. The use of organic fertilizers such as manure, compost and urban organic waste led to an increase of soil organic carbon of $0.16,0.81$ et $0.42 \mathrm{MgC} \mathrm{ha}^{-1}$ year $^{-1}$ respectively, but these increases were not significant due to the great variability of soil organic carbon values. Results confirm the capacity of some climate smart practices to
\end{abstract}

\footnotetext{
$\overline{\text { *Auteur de correspondance }}$ : tantely.razafimbelo@gmail.com
} 
store more carbon in the soil and hence to compensate for greenhouse gases emissions. However, the storage potentiality is quite different according to the practice and its spatial extent.

Keywords: adaptation / mitigation / climate change / organic matter / carbon sequestration / Madagascar

\section{Introduction}

Madagascar est reconnue pour sa grande diversité biophysique et sa richesse en termes de ressources naturelles, mais aussi pour sa grande pauvreté. L'agriculture tient une place importante dans la vie économique du pays et en fait la première solution pour pallier l'insécurité alimentaire et améliorer le revenu des paysans. Les pratiques traditionnelles prédominantes, basées sur la culture de riz sur brûlis aux dépens des forêts, figurent parmi les premières causes de déforestation dans les zones à proximité des forêts. Ces pratiques peuvent entraîner une dégradation importante des sols si des mesures de protection contre l'érosion ou des mesures de gestion durable de la fertilité ne sont pas entreprises (Kleinman et al., 1995; Gay-des-Combes et al., 2017). Dans les milieux cultivés sur les Hautes Terres ou en dehors des milieux forestiers, les pratiques agricoles sont basées sur les cultures pluviales, avec un labour du sol manuel ou par traction animale, une très faible utilisation de la fertilisation minérale et un peu de fertilisation organique au niveau des parcelles rizicoles et des cultures maraîchères de bas-fonds. Les résidus de récoltes sont soit exportés pour nourrir les animaux, soit utilisés dans les étables pour servir de litière. Le fumier ainsi élaboré est généralement restitué en grande partie sur les rizières. Les parcelles sur tanety (ou versants en cultures pluviales) sont très rarement fertilisées ou amendées. Ces pratiques traditionnelles conduisent à une diminution de la fertilité des sols et à leur dégradation et entraînent souvent l'abandon des terres par les paysans, qui défrichent de nouvelles terres.

Pour éviter la dégradation de la fertilité des sols et assurer durablement la production agricole, différentes initiatives ont été mises en place afin d'aider les paysans à maintenir durablement leur productivité et à faire face aux changements climatiques. Parmi ces initiatives, on peut citer la mise en place de l'agroécologie et de la "Climate Smart Agriculture» ou agriculture climato-intelligente (Lipper et al., 2014; Paustian et al., 2016), dans les espaces cultivés et autour des zones forestières.

L'agriculture intelligente face au climat a trois objectifs principaux: la sécurité alimentaire par une augmentation durable de la productivité et des revenus agricoles; 1'adaptation et le renforcement de la résilience face aux impacts des changements climatiques; et l'atténuation de l'effet de serre par la réduction et/ou la suppression des émissions de gaz à effet de serre (GES) et la séquestration du carbone (FAO, 2010). L'agriculture climato-intelligente est mentionnée dans le cadre de l'Initiative $4 \%$ ( 4 pour mille) qui a pour objectif d'augmenter les stocks globaux de carbone des sols de $0,4 \%$ par an pour compenser les émissions globales de GES d'origine humaine (Lal, 2016).

Parmi ces pratiques agricoles climato-intelligentes, on peut citer l'agriculture de conservation, l'agroforesterie, l'amélioration des pratiques rizicoles, les rotations et/ou associations de cultures ou l'utilisation d'intrants organiques. L'agriculture de conservation est une pratique basée sur trois piliers : réduction ou suppression du travail du sol, couverture permanente du sol et rotations diversifiées. À Madagascar, l'agriculture de conservation a été introduite depuis 1991 sur les Hautes Terres et a ensuite diffusé dans les autres zones. Le développement de cette pratique s'est heurté à différentes contraintes techniques et sociales, mais toutefois, elle a été adoptée - et adaptée - par les paysans les plus convaincus. L'agroforesterie est l'association d'arbres à des activités agricoles; elle est présente dans l'île sous différentes formes avec deux types dominants: une arboriculture fruitière avec des cultures vivrières sur les Hautes Terres, et des cultures de rente (girofliers, arbre à litchis, etc.) associées à des cultures vivrières sur les côtes. L'épandage du fumier est généralement pratiqué par les paysans sur les parcelles rizicoles, car la culture de riz est pour eux prioritaire, le riz étant l'aliment de base à Madagascar. L'épandage de compost est également pratiqué de plus en plus couramment dans les milieux ruraux.

La séquestration de carbone par ces pratiques agricoles climato-intelligentes peut être importante ou non selon les capacités de la pratique:

- à augmenter les apports de carbone au sol à travers les résidus ou les intrants organiques;

- à réduire les pertes de carbone par minéralisation à travers la suppression du labour, l'amélioration de la stabilité structurale du sol ou la modification de la qualité des résidus organiques restitués.

Lal et Bruce (1999), dans une revue bibliographique regroupant plusieurs pratiques agricoles durables et pouvant être classées parmi les pratiques climato-intelligentes, ont rapporté des stockages annuels allant de 0 à plus de $2 \mathrm{MgC}$ $\mathrm{ha}^{-1} \mathrm{an}^{-1}$ (Lal et Bruce, 1999), qui varient en fonction de différents paramètres comme le climat, l'altitude, le type de sol, et les types de pratiques mis en œuvre. À titre d'exemple, l'utilisation du fumier peut augmenter le stock de carbone du sol de 0,02 à $0,08 \%$ an $^{-1}$ en Inde (Nambiar, 1995), et de 0,03 à $0,7 \% \mathrm{an}^{-1}$ en Afrique de l'Ouest (Smith et Naazie, 1998).

Deux types d'approches peuvent être utilisés pour évaluer l'effet d'une pratique donnée sur le stockage de carbone. L'approche diachronique consiste à mesurer sur une même parcelle le stockage de carbone entre un temps t0 et un temps $\mathrm{x}$. Cette méthode serait la méthode d'évaluation la plus pertinente mais sa mise en œuvre est limitée par le fait que l'observateur doit attendre plusieurs longues années avant de pouvoir évaluer la quantité de carbone séquestré (Bernoux et al., 2005). L'approche synchronique consiste à comparer, à un instant donné, le stock de carbone d'une parcelle sous une pratique alternative séquestrante menée pendant $\mathrm{x}$ années à celui d'une parcelle en mode de gestion conventionnel n'ayant pas subi de modifications de gestion et supposée représenter le temps t0 de la parcelle en système séquestrant. Ce type d'approche peut induire une surestimation de la séquestration, qui pourrait être 
Tableau 1. Systèmes étudiés en agriculture de conservation (stations expérimentales).

Table 1. Studied conservation agriculture systems (experimental stations).

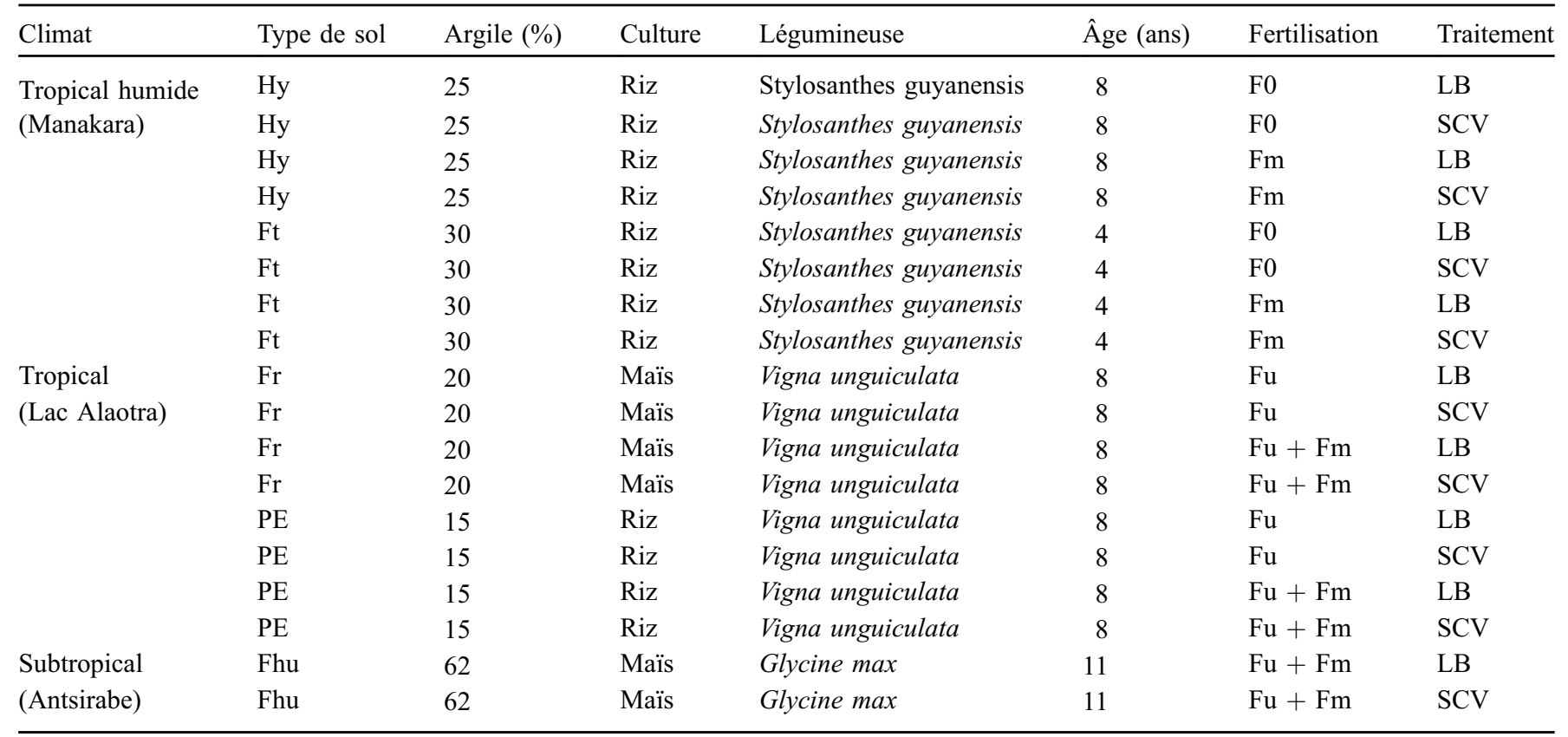

Hy : sol hydromorphe ou gleysol; Ft : sol ferrallitique typique ou ferralsol; Fr : sol ferrallitique rajeuni ou cambic ferralsol; PE : sol peu évolué d'apport ou fluvisol; Fhu : sol ferrallitique humifère ou ferralsol; F0 : sans fertilisation; Fu : fumier à raison de $5 \mathrm{Mg} \mathrm{Ma}^{-1}$; Fm : fertilisation minérale à raison de $62,5 \mathrm{~kg}$ de N, 14,5 $\mathrm{kg}$ de $\mathrm{P}$ et 19,9 $\mathrm{kg}$ de $\mathrm{K}$ par hectare et par an; LB : labour (référence) ; SCV: systèmes en semis direct avec couverture végétale.

due à une diminution accrue des stocks de carbone pour la parcelle en gestion conventionnelle due à des pertes de carbone par érosion (Bernoux et al., 2005). Mais c'est la seule approche qui permette d'évaluer la séquestration de carbone par des pratiques alternatives déjà mises en place en milieu paysan.

Cette étude a ainsi pour objectif de donner un aperçu des potentiels de séquestration additionnelle de carbone de quelques pratiques agricoles climato-intelligentes mises en œuvre à Madagascar.

\section{Matériels et méthodes}

\subsection{Sites et traitements étudiés}

L'étude est effectuée dans différentes régions de Madagascar à travers la sélection des pratiques climato-intelligentes existantes. Elle est ainsi effectuée, selon les localisations des pratiques étudiées, sur la Côte-Est, les Hautes Terres et le SudEst, avec des conditions pédoclimatiques contrastées.

Trois principales pratiques ont été étudiées: l'agriculture de conservation, l'agroforesterie et l'utilisation des intrants agricoles.

Deux types de dispositifs ont été sélectionnés pour l'étude :

- des systèmes en milieu paysan (cas de l'agroforesterie);

- des dispositifs expérimentaux (cas de l'agriculture de conservation, cas de l'utilisation des intrants organiques).

\subsubsection{L'agriculture de conservation (AC)}

Les systèmes étudiés font partie des anciens dispositifs expérimentaux en station de l'Organisation non gouverne- mentale (ONG) TAny sy FAmpandrosoana (TAFA). Ils comportent des systèmes de culture conduits en semis direct avec couverture végétale, appelés systèmes SCV. Ces dispositifs ont été installés sur des jachères de bozaka (Aristida sp.) âgées de plus de 10 ans. Plusieurs systèmes $\mathrm{SCV}$, en non labour, associant céréales (riz ou maïs) et légumineuses (niébé, soja, stylosanthes), avec différents niveaux de fertilisation (sans fertilisation ou F0, fertilisation minérale ou Fm, fumier ou $\mathrm{Fu}$, fertilisation minérale et organique ou $\mathrm{Fm}+\mathrm{Fu}$ ), ont été sélectionnés (Tab. 1). Différentes conditions pédoclimatiques ont été prises en compte, allant du climat tropical humide $\left(\mathrm{T}=19^{\circ} \mathrm{C}\right.$, $\mathrm{P}=2500 \mathrm{~mm}$ à Manakara $)$ au climat tropical $\left(\mathrm{T}=20^{\circ} \mathrm{C}\right.$, $\mathrm{P}=1200 \mathrm{~mm}$ au Lac Alaotra) et tropical d'altitude ( $\mathrm{T}=17^{\circ} \mathrm{C}, \mathrm{P}=1300 \mathrm{~mm}$ dans les Hautes Terres), ainsi que des sols de différents types allant des sols sableux aux sols argileux (Tab. 1). Des systèmes analogues mais labourés ont été pris comme référence (Tab. 1). Chaque site expérimental comprenait trois parcelles de répétition par traitement.

\subsubsection{L'agroforesterie (AF)}

Les systèmes étudiés sont des systèmes agroforestiers en milieu paysan, associant des girofliers à des cultures annuelles (riz associé au manioc), dans l'écorégion Est à proximité de la forêt humide de l'est de Madagascar $\left(\mathrm{T}=25^{\circ} \mathrm{C}, \mathrm{P}=3000 \mathrm{~mm}\right)$, dans la région d'Analanjirofo (commune d'Ambodimanga) et dans la Région d'Atsinanana, sur des sols ferrallitiques (ferralsol) sablo-argileux $(40-50 \%$ d'argile; $25-45 \%$ de sables). Deux systèmes agroforestiers, âgés de 24 ans, comprenant des parcelles paysannes de girofliers associés à 
Tableau 2. Systèmes étudiés en agroforesterie paysanne.

Table 2. Studied agroforestry systems on farmers plots.

\begin{tabular}{|c|c|c|c|c|c|}
\hline Traitement & Commune & Point GPS & Âge d'exploitation ${ }^{*}$ & Cycle cultural & Argile $(\%)$ \\
\hline Abattis-brûlis & Ambodimanga II & $\begin{array}{l}17^{\circ} 19^{\prime} 49,0^{\prime \prime} \mathrm{S} ; 49^{\circ} 20^{\prime} 39,3^{\prime \prime} \mathrm{E} \\
18^{\circ} 19^{\prime} 49,0^{\prime \prime} \mathrm{S} ; 49^{\circ} 20^{\prime} 39,3^{\prime \prime} \mathrm{E} \\
17^{\circ} 19^{\prime} 52,7{ }^{\prime \prime S} ; 49^{\circ} 20^{\prime} 51,9^{\prime \prime} \mathrm{E} \\
17^{\circ} 19^{\prime} 51,6{ }^{\prime} \mathrm{S} ; 49^{\circ} 20^{\prime} 51,5^{\prime \prime E}\end{array}$ & 25 ans & $\begin{array}{l}\text { Riz/jachère } 2 \text { ans /culture } \\
\text { de manioc en abattis } \\
\text { brûlis }\end{array}$ & 49 \\
\hline Abattis & Ambodimanga II & 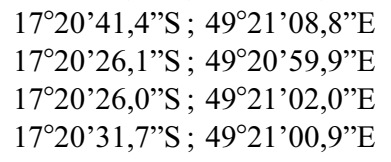 & 25 ans & $\begin{array}{l}\text { Jachère, culture associée } \\
\text { riz/manioc ou maïs en } \\
\text { abattis }\end{array}$ & 44 \\
\hline \multicolumn{6}{|l|}{ Agroforesterie } \\
\hline $\begin{array}{l}\text { AFS (Agroforesterie } \\
\text { simple) }\end{array}$ & Ambodimanga II & 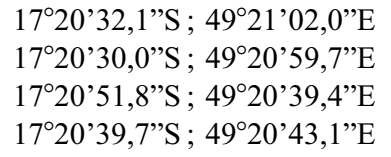 & $\begin{array}{l}\text { Âge des girofliers: } \\
20 \text { à } 40 \text { ans } \\
24 \text { ans de cultures } \\
\text { en association }\end{array}$ & $\begin{array}{l}\text { Giroflier associé à Riz/ } \\
\text { manioc } \\
\text { Densité des girofliers : } \\
<200 \text { arbres ha }^{-1}\end{array}$ & 47 \\
\hline
\end{tabular}

\footnotetext{
* À dires d'exploitants.
}

du riz et du manioc, ont été distingués suivant la densité des girofliers (Tab. 2) :

- les systèmes agroforestiers complexes (AFC), où la densité de girofliers est supérieure à 200 arbres par hectare ;

- des systèmes agroforestiers simples (AFS), où la densité de girofliers est inférieure à 200 arbres par hectare.

Les girofliers sont âgés de 20 à 40 ans. Les cultures annuelles ont été mises en place par les paysans, il y a 24 ans.

Les systèmes de référence (Tab. 2) sont des parcelles paysannes utilisées :

- en systèmes sous couverture végétale (Abattis), où la culture de riz est effectuée sans brûlis et sans labour;

- en culture conventionnelle de riz, c'est-à-dire la culture de riz sur brûlis (Abattis-Brûlis).

Pour ces deux systèmes de référence, la conduite de culture comprend des cycles de culture de 5-6ans composés de 2 années de jachère, suivies de deux années de culture de riz seul, d'une année ou non de culture de riz associée au manioc en fonction de la diminution du rendement du riz constaté par le paysan et d'une année de culture de manioc. L'ordre de succession des cycles n'est pas complètement défini d'un cycle à un autre. Ces systèmes ont été conduits comme tels depuis 25 années successives sur une défriche forestière. Pour chaque système (AFS, AFC, Abattis, Abattis-brûlis), quatre parcelles paysannes ont été échantillonnées en 2013.

\subsubsection{L'utilisation des intrants organiques}

L'étude a été effectuée sur une station d'expérimentation agronomique à Lazaina, Antananarivo $\left(\mathrm{T}=19^{\circ} \mathrm{C}, \mathrm{P}=1350 \mathrm{~mm}\right)$,
Tableau 3. Systèmes étudiés utilisant des intrants organiques. Table 3. Treatments using organic fertilizer inputs.

\begin{tabular}{lllll}
\hline Traitement & Intrants organiques & Culture & $\begin{array}{l}\text { Âge } \\
\text { d'exploitation }\end{array}$ & $\begin{array}{l}\text { Argile } \\
(\%)\end{array}$ \\
\hline Témoin & 0 & Maïs & 6 ans & 40 \\
Fumier & Fumier de bovin & Maïs & 6 ans & 40 \\
Compost & $\begin{array}{l}\text { Compost de résidus } \\
\text { végétaux }\end{array}$ & Maïs & 6 ans & 40 \\
Terreau & $\begin{array}{l}\text { Compost de déchets } \\
\text { urbains }\end{array}$ & Maïs & 6 ans & 40 \\
& & & \\
\hline
\end{tabular}

sur un sol ferrallitique (ferralsol) argileux (40\% d'argile) sous jachère depuis plus de 20 ans.

Trois types de matière organique ont été étudiés (Tab. 3):

- le fumier;

- le compost (fabriqué à partir de résidus végétaux);

- le terreau (compost élaboré à partir de déchets organiques urbains).

Les trois types d'intrants ont été testés pendant 6 ans sur une culture de maïs. Ils ont été comparés à un traitement sans apports organiques.

Les doses de compost et de terreau ont été ajustées à raison de $1000 \mathrm{~kg} \mathrm{C} \mathrm{ha}^{-1}$ d'équivalent carbone, de telle sorte que les doses de carbone pour les trois intrants soient équivalentes. Chaque traitement a été répété sur 4 blocs. Les stocks de carbone au début (2006) et à la fin (2012) de l'expérimentation ont été mesurés, afin de calculer le stockage induit par chaque type de matière organique apportée. 


\subsection{Prélèvements de sol et mesure des stocks de carbone}

Pour chaque traitement, des sols ont été prélevés sur chacune des parcelles élémentaires avec des cylindres de $259 \mathrm{~cm}^{3}$ pour les profondeurs de $0-5,5-10 \mathrm{~cm}$, et avec des cylindres de $513 \mathrm{~cm}^{3}$ pour la profondeur $10-20 \mathrm{~cm}$.

Pour mesurer la densité apparente, le sol contenu dans chaque cylindre est directement pesé et une aliquote de $50 \mathrm{~g}$ est prélevée, mise à l'étuve à $105^{\circ} \mathrm{C}$ pendant 48 heures et ensuite pesée afin d'obtenir le poids sec permettant le calcul de la densité apparente. Le reste des échantillons est séché à l'air, tamisé à $2 \mathrm{~mm}$, puis broyé à $0,2 \mathrm{~mm}$ pour l'analyse de la teneur en carbone organique.

La teneur en carbone organique est mesurée par combustion par voie humide suivant la méthode Walkley et Black (1934) suite à une oxydation de la matière organique par le bichromate de potassium.

Les stocks de carbone sont en Mégagrammes de carbone (tonnes) par hectare $\left(\mathrm{Mg} \mathrm{C} \mathrm{ha}^{-1}\right)$ et ont été calculés à partir de la teneur en carbone du sol (C, exprimé en $\left.\mathrm{g} \mathrm{kg}^{-1} \mathrm{sol}\right)$, de la densité apparente du sol ( $\mathrm{Da}$, exprimé en $\mathrm{g} \mathrm{cm}^{-3}$ ), de l'épaisseur de l'horizon prélevé (e, exprimé en $\mathrm{cm}$ ) et du

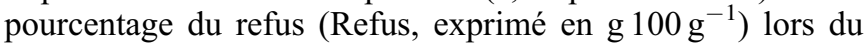
tamisage à $2 \mathrm{~mm}$, selon la formule ci-dessous :

Stock $\mathrm{C}=(\mathrm{C} \times(100-$ Refus $) \mathrm{Da} \times \mathrm{e}) / 10$.

Les différences de variation de stocks (exprimés en $\mathrm{Mg}$ $\mathrm{C} \mathrm{ha}{ }^{-1} \mathrm{an}^{-1}$ ) sont obtenues par la différence entre les stocks de carbone de la pratique alternative avec les pratiques de référence, ramenée à la durée de la mise en pratique. Elles sont ensuite exprimées en \%o par an.

À cause des différences en termes de densité apparente, afin de permettre une juste comparaison entre les traitements d'une même pratique, les stocks ont ensuite été calculés à masse équivalente de 1'horizon 0-20 cm (Ellert et Bettany, 1995).

L'approche synchronique consistant à comparer, à un instant donné, le stock de carbone d'une parcelle sous une pratique alternative séquestrante menée pendant $\mathrm{x}$ années à celui d'une parcelle en mode de gestion conventionnel n'ayant pas subi de modifications de gestion et supposée représenter le temps t0 de la parcelle en système séquestrant, a été utilisée pour évaluer l'effet de l'agriculture de conservation et l'agroforesterie sur les stocks de carbone, du fait de l'indisponibilité des valeurs de stocks de $\mathrm{C}$ au temps initial.

L'approche diachronique, consistant à mesurer sur une même parcelle le stockage de carbone entre un temps t0 (mise en place du dispositif) et un temps $x$, a été utilisée pour évaluer l'effet de l'utilisation des intrants organiques.

\subsection{Analyse statistique}

Les moyennes de stockage de carbone ont été calculées afin de montrer les différences entre les traitements. Dans cette étude, en raison des grandes différences entre les dispositifs (parcelles expérimentales et parcelles paysannes), les analyses statistiques ont été effectuées pour chaque pratique agricole climato-intelligente étudiée (agriculture de conservation, agroforesterie et utilisation des intrants agricoles), sans effectuer de comparaisons statistiques entre les différents types de pratiques. Pour les pratiques sur dispositifs expérimentaux (agriculture expérimentale et utilisation des intrants agricoles), l'analyse de variance (ANOVA) a été utilisée pour analyser l'effet des traitements. L'ANOVA a été suivie d'un test de comparaison des moyennes des stocks de carbone entre les différentes pratiques à $p<0,05$ (test de Student Newman Keuls). Pour l'agroforesterie située dans des parcelles paysannes, les effets des traitements sur les stocks de carbone peuvent être affectés par la grande variabilité spatiale du sol due à la répartition des parcelles paysannes. Ainsi, les stocks de carbone ont été soumis au test de comparaison des moyennes ( $p<0,05$; test de Student Newman Keuls) pour tester les éventuels effets des pratiques.

\section{Résultats et discussions}

\subsection{Le stockage de carbone par l'agriculture de conservation}

Les valeurs des stocks observées pour les différentes situations étudiées sont très variables (Tab. 4). Les différences entre pratiques varient de 0 jusqu'à des différences très significatives de $1,82 \mathrm{MgCha}^{-1} \mathrm{an}^{-1}$. D'une manière générale, il est difficile, à partir de ces résultats, d'observer un effet climat sur le stockage du carbone, compte tenu de la grande variabilité des valeurs observées. Des valeurs légèrement plus élevées sont toutefois observées pour le climat tropical humide. Une importante différence de stocks de $1,82 \mathrm{MgC}$ $\mathrm{ha}^{-1} \mathrm{an}^{-1}$ est observée entre le traitement Riz-Stylosanthes en agriculture de conservation et le traitement Riz-Stylosanthes en labour conventionnel pour le sol hydromorphe (Hy) avec $25 \%$ d'argile. Cela peut s'expliquer par le fait que dans ces conditions d'hydromorphie, la matière organique peut s'accumuler car sa minéralisation est ralentie du fait de l'absence d'oxygène. Quant aux autres systèmes, en dehors d'un système Riz-Stylosanthes en climat tropical humide qui donne une différence de stockage nulle avec le labour conventionnel, les pratiques d'agriculture de conservation étudiées ici permettent d'augmenter le stock de carbone du sol avec des valeurs moyennes de $0,58 \pm 0,51 \mathrm{MgC} \mathrm{ha}^{-1} \mathrm{an}^{-1}$ pour une profondeur équivalente de $20 \mathrm{~cm}$. Ces valeurs sont assez cohérentes, voire plus élevées que celles observées dans la littérature. En Afrique subsaharienne, une méta-analyse effectuée par Powlson et al. (2016) rapporte des valeurs de stockage de $0,54 \mathrm{MgCha}^{-1} \mathrm{an}^{-1}$ pour l'agriculture de conservation. Les augmentations de stock de carbone par ces systèmes sont liées essentiellement à la quantité importante de résidus organiques restituée par les cultures et les plantes de couverture, mais aussi à la suppression du labour, qui ralentit la minéralisation de la matière organique du sol et entraîne une redistribution du carbone du sol (Powlson et al., 2014). Une méta-analyse effectuée par Poeplau et Don (2015) montre d'ailleurs que l'introduction de plantes de couverture dans la rotation culturale peut à elle seule générer une augmentation de stock de carbone de $0,32 \mathrm{MgCha}^{-1}$ an $^{-1}$ à $0-22 \mathrm{~cm}$ de profondeur pendant une période de 54 ans. Les valeurs de stocks de carbone sont souvent affectées d'une grande variabilité entraînant une difficulté d'évaluation des effets significatifs. Dans une revue bibliographique récente (Corbeels et al., à paraître), sur le potentiel de l'agriculture de conservation à atteindre un taux d'augmentation du carbone $\mathrm{du}$ sol de 4\% par an en Afrique subsaharienne, des valeurs 
Tableau 4. Stockage de carbone en $\mathrm{MgCha}^{-1} \mathrm{an}^{-1}$ par l'agriculture de conservation ( $n=3$, moyennes et écart-types). Table 4. Soil carbon storage, in $\mathrm{MgCha}^{-1}$ year $^{-1}$, for conservation agriculture.

\begin{tabular}{|c|c|c|c|c|c|c|}
\hline Climat & Type de sol & Argile (\%) & Culture & Légumineuse & Fertilisation & Stockage C \\
\hline \multirow{2}{*}{ Tropical humide } & Hy & 25 & Riz & Stylosanthes guyanensis & $\mathrm{Fm}$ & $1,816^{*}$ \\
\hline & $\mathrm{Ft}$ & 30 & Riz & Stylosanthes guyanensis & $\mathrm{Fm}$ & 0 \\
\hline \multirow[t]{3}{*}{ Tropical } & $\mathrm{Fr}$ & 20 & Maïs & Vigna unguiculata & $\mathrm{Fu}$ & 0,137 \\
\hline & $\mathrm{Fr}$ & 20 & Maïs & Vigna unguiculata & $\mathrm{Fu}+\mathrm{Fm}$ & 0,22 \\
\hline & $\mathrm{PE}$ & 15 & Riz & Vigna unguiculata & $\mathrm{Fu}+\mathrm{Fm}$ & 0,6 \\
\hline Subtropical & Fhu & 62 & Maïs & Glycine $\max$ & $\mathrm{Fu}+\mathrm{Fm}$ & 0,327 \\
\hline
\end{tabular}

Hy : sol hydromorphe ou gleysol; Ft : sol ferrallitique typique ou ferralsol ; Fr: sol ferrallitique rajeuni ou cambic ferralsol ; PE : sol peu évolué d'apport ou fluvisol ; Fhu : sol ferrallitique humifère ou ferralsol ; F0 : sans fertilisation; Fu : fumier à raison de $5 \mathrm{Mg} \mathrm{MS} \mathrm{ha}{ }^{-1}$; Fm : fertilisation minérale à raison de $62,5 \mathrm{~kg}$ de $\mathrm{N}, 14,5 \mathrm{~kg}$ de $\mathrm{P}$ et $19,9 \mathrm{~kg}$ de $\mathrm{K}$ par hectare par an.

${ }^{*}$ Stockage significatif: les stocks initiaux (LB) et les stocks sous SCV sont significativement différents $(p<0,05)$.

extrêmement diverses de changement de stock de carbone ont été relevées, allant de $-10,2(0-30 \mathrm{~cm})$ à $+6,86(0-15 \mathrm{~cm})$ $\mathrm{MgC} \mathrm{ha}{ }^{-1} \mathrm{an}^{-1}$, ce qui représente des taux d'augmentation de -142 à $+450 \% \mathrm{an}^{-1}$. La dispersion de ces valeurs ne permet pas de conclure de manière significative sur le potentiel de l'agriculture de conservation à augmenter le carbone du sol en Afrique subsaharienne.

Quant à la fertilisation, elle agit d'une manière générale sur la biomasse produite par les cultures et ensuite restituée au sol (Dou et al., 2014). La fertilisation organique peut aussi agir sur la minéralisation du carbone du sol à travers la stimulation de l'activité microbienne qui décompose une fraction de carbone native du sol, connue également sous l'appelation «priming effect» (Chen et al., 2014; Bernard et al., 2012; Kuzyakov et al., 2000). Pour les systèmes étudiés ici, l'effet positif de la fertilisation n'est toutefois observé que pour le système RizStylosanthes du sol hydromorphe (Hy) $(p<0,05)$. Pour les sols en climat tropical, on observe une tendance d'effet de la fertilisation sur le stockage, mais elle est non significative. Il est également reporté que le stock de $\mathrm{C}$ n'augmente pas indéfiniment mais tend vers une valeur d'équilibre (Johnston et al., 2009; Powlson et al., 2012; Smith, 2014) pour donner une représentation curvilinéaire de cette accumulation de $\mathrm{C}$ avec le temps.

\subsection{Le stockage de carbone par les systèmes agroforestiers de l'est de Madagascar}

Les stocks de carbone du sol observés pour les 4 traitements sont très différents $(p<0,05)$. Ils sont significativement plus faibles pour les systèmes en riz sur brûlis par rapport aux autres traitements (Fig. 1). Ainsi, par rapport aux cultures sur brûlis, la culture du riz sur abattis permet de stocker $0,41 \mathrm{MgC} \mathrm{ha}^{-1} \mathrm{an}^{-1}$, tandis que les systèmes agroforestiers simples et complexes permettent de stocker respectivement 0,68 et $0,65 \mathrm{Mg} \mathrm{Cha}^{-1}$ an $^{-1}$ après 24 ans, soit respectivement une augmentation de 8,5 et $8,9 \%$ par an. Nair et al. (2009) suggèrent des valeurs de séquestration de carbone dans le sol allant de 0,1 à $0,25 \mathrm{MgCha}^{-1} \mathrm{an}^{-1}$

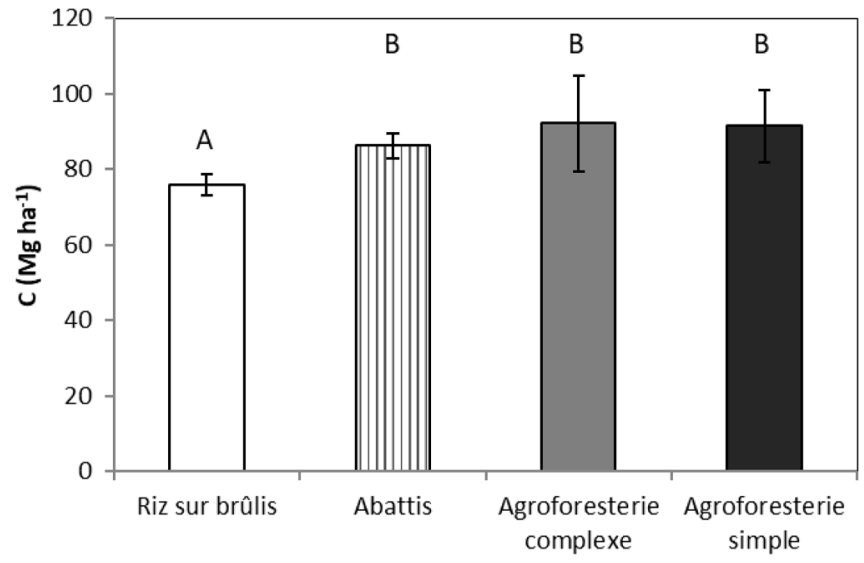

Fig. 1. Stocks de carbone du sol pour des systèmes agroforestiers à base de girofliers dans la partie Est de Madagascar (moyennes et écart-types, $n=4)$.

Fig. 1. Soil carbon stocks in cloves agroforestry systems, East of Madagascar (means and standard deviation, $\mathrm{n}=4$ ).

pendant 10 ans pour des systèmes agroforestiers en climat tropical humide. Albrecht et al. (données non publiées), dans le cadre d'un projet sur l'agroforesterie au Kenya, suggèrent des valeurs de $0,27 \mathrm{MgCha}^{-1} \mathrm{an}^{-1}$. Les valeurs observées à Madagascar sont ainsi relativement élevées. À Madagascar, le stockage de carbone par les systèmes agroforestiers peut être très différent du fait de la diversité des systèmes possibles: arbres et cultures annuelles mélangées (cas de cette étude), arbres et cultures annuelles mélangées suivant des dispositions en ligne, arbres disposés autour de la parcelle cultivée en cultures annuelles, mélange d'arbres de différents types (arbres fruitiers et arbres d'ombrage) formant différentes strates. En Afrique subsaharienne, Corbeels et al., 2018 indiquent des variations de stocks allant de $-9,6(0-40 \mathrm{~cm})$ à $+14 \mathrm{Mg} \mathrm{C}$ $\mathrm{ha}^{-1} \mathrm{an}^{-1}(0-100 \mathrm{~cm})$, ce qui représente des taux d'augmentation de -159 à $+194 \%$ an ${ }^{-1}$. Des taux de stockage du carbone significativement supérieurs à $4 \%$ ont été trouvés uniquement pour les jachères agroforestières et les systèmes agroforestiers 


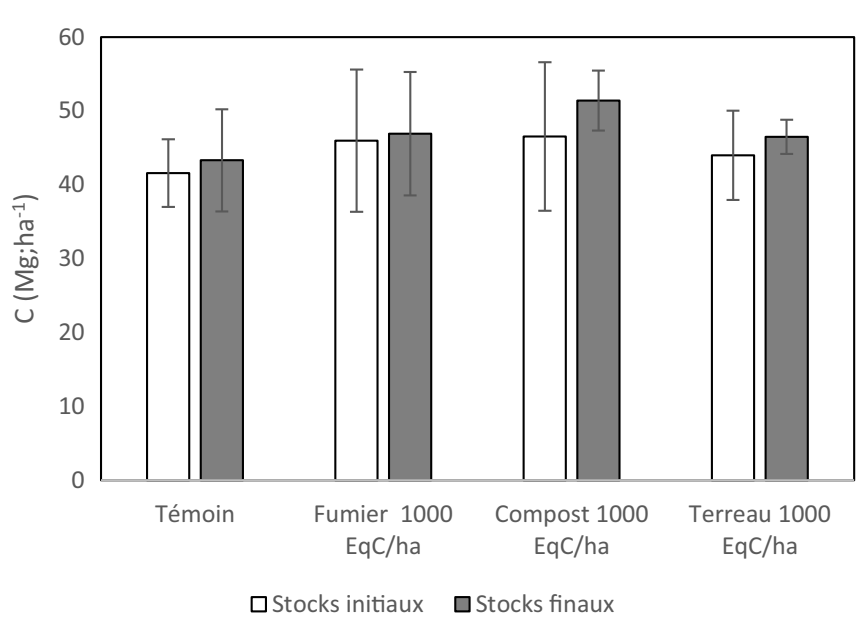

Fig. 2. Stocks de carbone du sol pour des traitements utilisant des intrants organiques : fumier, compost et terreau (moyennes et écarttypes, $n=4)$.

Fig. 2. Soil carbon stocks in treatments using organic inputs: manure, compost and urban organic waste (means and standard deviation, $\mathrm{n}=4$ ).

multistrates, pas pour les cultures en couloirs ni les arbres dispersés dans les champs. En plus du stockage dans le sol, les systèmes agroforestiers permettent également de stocker du carbone dans la biomasse par l'intermédiaire des arbres. Pour les systèmes étudiés ici, ces séquestrations ont été respectivement de 0,47 et $0,63 \mathrm{MgCha}^{-1} \mathrm{an}^{-1}$ pour les systèmes en agroforesterie simple et complexe (Ramiandrisoa, 2009). Ces systèmes peuvent ainsi présenter un fort potentiel de séquestration de carbone si on prend en compte les deux compartiments, sol et plante.

\subsection{Le stockage de carbone par l'utilisation des intrants organiques}

Les valeurs observées avec l'utilisation d'intrants organiques montrent des augmentations du stock de carbone non significatives pour l'ensemble des traitements après 6 ans (Fig. 2). Cela est dû principalement à la variabilité du stock de carbone pour le dispositif étudié. En effet, le dispositif étant mis en place sur un versant, la variabilité spatiale induite par les différences entre blocs de répétitions a été importante. Toutefois, malgré l'absence d'un effet significatif, une tendance à l'augmentation est observée et il n'y a pas de diminution du stock induite par la mise en place des traitements. Ces résultats impliquent que l'utilisation des intrants organiques permet de maintenir la teneur en carbone du sol et de retenir ainsi durablement sa fertilité. Les stockages de carbone sont respectivement de $0,16,0,81$ et $0,42 \mathrm{MgC}$ $\mathrm{ha}^{-1} \mathrm{an}^{-1}$ pour les traitements utilisant du fumier, du compost et du terreau, alors qu'il est de $0,288 \mathrm{Mg} \mathrm{Cha}^{-1} \mathrm{an}^{-1}$ pour le traitement témoin; les stocks initiaux étant respectivement de $46,0,46,6$ et $44,0 \mathrm{MgC} \mathrm{ha}^{-1}$ et les stocks finaux de 46,9, 51,4 et $46,5 \mathrm{MgC} \mathrm{ha}^{-1}$ pour les traitements utilisant du fumier, du compost et du terreau. L'utilisation du compost peut ainsi induire une accumulation plus importante de carbone du fait de sa maturité et de son degré d'humification avancé par rapport aux autres intrants organiques. En effet, la qualité et la quantité des intrants organiques apportés affectent l'accumulation du carbone dans le sol, particulièrement dans les sols présentant des déficits de saturation en carbone élevés, comme les sols tropicaux (Fujisaki et al., 2018).

\section{Conclusion}

Cette étude a mis en évidence le potentiel de quelques pratiques agricoles climato-intelligentes existant déjà à Madagascar pour augmenter ou maintenir le carbone organique du sol. Elle met toutefois en exergue la difficulté d'évaluation de l'impact de ces pratiques sur le carbone du sol, entre autres du fait de l'importance de la variabilité spatiale, dont il faut tenir compte, car cette variabilité masque dans certaines situations l'effet des pratiques. Il est ainsi important de mettre en place des dispositifs expérimentaux efficaces afin de pouvoir effectuer une quantification précise de l'effet de ces pratiques sur le carbone du sol en milieu paysan tropical.

\section{Références}

Bernard L, Chapuis-Lardy L, Razafimbelo T, Razafindrakoto M, Pablo A-L, Legname E, et al. 2012. Endogeic earthworms shape bacterial functional communities and affect organic matter mineralization in a tropical soil. ISME Journal 6: 213-22. DOI: 10.1038/ismej.2011.87.

Bernoux M, Feller C, Cerri CC, Eschenbrenner V, Cerri CEP. 2005. Soil carbon sequestration. In: Roose EJ, Lal R, Feller C, Barthès B, Stewart BA (eds). Soil erosion and carbon dynamics. Advances in Soil Science. CRC, Taylor and Francis.

Chen R, Senbayram M, Blagodatsky S, Myachina O, Dittert K, Lin X, et al. 2014. Soil C and $\mathrm{N}$ availability determine the priming effect: microbial $\mathrm{N}$ mining and stoichiometric decomposition theories. Global Change Biology 20: 2356-2367. DOI: 10.1111/ gcb.12475.

Corbeels M, Cardinael R, Naudin K, Guibert H, Torquebiau E. 2018. The 4 per 1000 goal and soil carbon storage under agroforestry and conservation agriculture systems in sub-Saharan Africa. Soil and Tillage Research. DOI: 10.1016/j.still.2018.02.015.

Dou F, Wight JP, Wilson LT, Storlien JO, Hons FM. 2014. Simulation of biomass yield and soil organic carbon under bioenergy sorghum production. PLOS ONE 9: e115598. DOI: 10.1371/journal. pone. 0115598 .

Ellert BH, Bettany JR. 1995. Calculation of organic matter and nutrients stored in soils under contrasting management regimes. Canadian Journal of Soil Science 75: 529-538.

FAO. 2010. "Climate-Smart" Agriculture. Policies, practices and financing for food security, adaptation and mitigation. Rome, Italie: FAO, 40 p.

Fujisaki K, Chevallier T, Chapuis-Lardy L, Albrecht A, Razafimbelo T, Masse D, et al. 2018. Soil carbon stock changes in tropical croplands are mainly driven by carbon inputs: a synthesis. Agriculture, Ecosystems and Environment 259: 147-158.

Gay-des-Combes JM, Robroek BJM, Herve D, Guillaume T, Pistocchi C, Mills RTE, et al. 2017. Slash-and-burn agriculture and tropical cyclone activity in Madagascar: implication for soil fertility dynamics and corn performance. Agriculture Ecosystems \& Environment 239: 207-218. 
Johnston AE, Poulton PR, Coleman K. 2009. Soil organic matter: its importance in sustainable agriculture and carbon dioxide fluxes. Advances in Agronomy 101: 2-57.

Kleinman PJA, Pimentel D, Bryant RB. 1995. The ecological sustainability of slash-and-burn agriculture. Agriculture, Ecosystems and Environment 52: 235-249.

Kuzyakov Y, Friedel JK, Stahr K. 2000. Review of mechanisms and quantification of priming effects. Soil Biology and Biochemistry 32: 1485-1498. DOI: 10.1016/S0038-0717(00)00084-5.

Lal R. 2016. Beyond COP 21: potential and challenges of the " 4 per Thousand" initiative. Journal of Soil and Water Conservation 71: 20A-25A.

Lal R, Bruce JP. 1999. The potential of world cropland soils to sequester $\mathrm{C}$ and mitigate the greenhouse effect. Environmental Science and Policy 2: 177-185.

Lipper L, Thornton P, Campbell BM, Baedeker T, Braimoh A, Bwalya M, et al. 2014. Climate-smart agriculture for food security. Nature Climate Change 4: 1068-1072.

Nair PKR, Nair VD, Kumar BM, Haile SC. 2009. Soil carbon sequestration in tropical agroforestry systems: a feasibility appraisal. Environmental Science \& Policy 12 (8): 1099-1111.

Nambiar KKM. 1995. Major cropping systems in India. In: Barnett V, Payne R, Steiner R (eds). Agricultural sustainability, economic, environmental and statistical considerations. Chichester: J. Wiley \& Sons.

Paustian K, Lehmann J, Ogle S, Reay D, Robertson GP, Smith P. 2016. Climate-smart soils. Nature 532: 49-57.
Poeplau C, Don A. 2015. Carbon sequestration in agricultural soils via cultivation of cover crops. A meta-analysis. Agriculture, Ecosystems and Environment 200: 33-41.

Powlson DS, Bhogal A, Chambers BJ, Macdonald AJ, Goulding KWT, Whitmore AP. 2012. The potential to increase soil carbon stocks through reduced tillage or organic material additions in England and Wales: a case study. Agriculture Ecosystems and Environment 146: 23-33. DOI: 10.1016/j.agee.2011.10.004.

Powlson DS, Stirling CM, Jat ML, Gerard BG, Palm CA, Sanchez PA, et al. 2014. Limited potential of no-till agriculture for climate change mitigation. Nature Climate Change 4: 678-683. DOI: 10.1038/nclimate2292.

Powlson DS, Stirling CM, Thierfelder C, Whited RP, Jate ML. 2016. Does conservation agriculture deliver climate change mitigation through soil carbon sequestration in tropical agro-ecosystems? Agriculture. Ecosystems and Environment 220: 164-174.

Ramiandrisoa S. 2009. Quantification du stock de carbone dans les systèmes agroforestiers en vue d'une simulation de projet financement de crédits carbone: cas de la Région Analanjirofo et Antsinanana. Mémoire d'ingénieur agronome. École supérieure des sciences agronomiques, Université d'Antananarivo.

Smith P. 2014. Do grasslands act as a perpetual sink for carbon? Global Change Biology 20: 2708-2711. DOI: 10.1111/gcb.12561.

Smith JW, Naazie A. 1998. The role of ruminant livestock in soil fertility management in sub-Saharan Africa. In : Lal R (ed). Soil quality and agricultural sustainability. Chelsea, MI: Ann Arbor Press, 214-221.

Citation de l'article : Razafimbelo TM, Andriamananjara A, Rafolisy T, Razakamanarivo H, Masse D, Blanchart E, Falinirina M-V, Bernard L, Ravonjiarison N, Albrecht A. 2018. Impact de l'agriculture climato-intelligente sur les stocks de carbone organique du sol à Madagascar. Cah. Agric. 27: 35001 . 